und vierte am längsten, fünfte kürzer als diese, zweite gleich der sechsten oder wenig kürzer. Zur Vergleichung seien nachfolgend auch noch die Maasse der drei Arten zusammengestellt:

C. baeticata: Flügel 58, Schwanz $55 \mathrm{Mm}$.

C. arundinacea: Flügel 64, Schwanz $55 \mathrm{Mm}$.

C. caffra: Flügel 64 , Schwanz $60 \mathrm{Mm}$. F AM. TURDIDAE.

25. Petrocincla saxatilis (L.).

413. juv. Bagamojo, März 1879.

26. Turdus libonyanus (Smith).

423. Mossambique, Mai 1879. Flügel 110, Schwanz 100, Mundspalte $24 \mathrm{Mm}$.

\title{
Beitrag zur Ornithologie des Werrathales in Thüringen von
}

G. Ruhmer, stud. rer. nat.

Schon seit einer Reihe von Jahren benutze ich meine Sommerferien zu einem Ausfluge nach Thüringen, wo ich in Barchfeld, einem Flecken an der Werra, unfern Bad Liebenstein mein Standquartier nehme. Meine Beobachtungen dort sind zwar hauptsächlich der Botanik gewidmet, ich habe aber auch stets den Vögeln einige Aufmerksamkeit geschenkt und will das, was ich beobachtet oder erfahren, hier mittheilen.

Das in Rede stehende Gebiet ist nur klein. Die Westgrenze bildet der Kamm eines Höhenzuges, der als östlicher Ausläufer der Rhön betrachtet werden muss; er liegt zwischen der Fulda und Werra.

Einen Namen dafür finde ich auf keiner Karte und auch in der Gegend selbst ist kein besonderer Name dafür gebräuchlich. Die Ostgrenze bildet der Thüringer Wald. Nach Norden mag eine gerade Linie von Etterwinden nach Salzungen die Grenze bilden. Nach Süden bin ich bis zum Rosabach vorgedrungen.

Das Gebiet enthält fast alle Bedingungen, die eine reich vertretene Ornis ermöglichen: Laub-, Nadel- und gemischten Wald mit Unterholz, Wiesen, Felder, einige Teiche und Seen, in der Mitte die mit Weidengebüsch eingefasste Werra.

Ich habe allerdings viele Vögel, die im Gebiet vorkommen 
mögen, noch nicht bemerkt, da ich wie gesagt nur im Sommer, resp. Herbst dort gewesen bin. Ich habe indessen auch nach Kräften Erkundigungen eingezogen und von vielen Seiten habe ich auch Zusendungen bemerkenswerther Vögel aus dem Gebiet erhalten.

Buteo vulgaris. Gemein. Auch ein schönes 오 der weissen Varietät erhielt ich vom Horst geschossen zugesandt.

Milvus regalis ist von jeher selten gewesen.

Falco peregrinus ist dagegen öfter beobachtet worden.

Astur palumbarius horstet nicht im Gebiet und ist eine nur seltene Erscheinung.

Astur nisus und Falco tinnunculus sind ungemein häufige Raubvögel.

Circus rufus hat nur einmal früher am Frauenbreitunger See gehorstet.

Bubo maximus soll noch (allerdings ausserhalb des Gebiets und bedeutend weiter südlich) in steilen Felsen an der Werrabahn horsten.

Strix brachyotus erhielt ich zweimal im Herbst (vermuthlich auf dem Zuge).

Strix aluco, noctua und flammea sind alle wohl häufig zu nennen. Von letzterer hauste ein Pärchen im Taubenschlage des Hofjägers Hergt in Altenbreitungen. Derselbe behauptet, die Eulen hätten eines Tages mehreren seiner Tauben die Köpfe abgebissen. Er schwur ihnen darauf Rache und schoss sie. Das Männchen besitzt ausgestopft der Lehrer Starke in Altenbreitungen, es ist dadurch auffallend, dass Brust und Bauch ganz weiss gefärbt sind.

Lanius excubitor und Lanius collurio sah ich allenthalben.

Lanius ruficeps. An der Liebensteiner Chaussée sah ich vier junge Vögel, die jedenfalls einem Neste entstammten.

Garrulus glandarius sehr gemein.

Nucifraga caryocatactes hat sich im Herbst vorigen Jahres bei Barchfeld gezeigt, konnte aber nicht erlegt werden.

Pica caudata, Corvus monedula und Corvus corone sind Brutvögel und in grosser Zahl anzutreffen. Der Abendversammlungsort der letzteren ist der Dönnersche Berg, eine mässige, meist unbebaute Erhebung; von demselben begeben sie sich auf den grossen Blessberg und die Hunnkuppe zur Nachtruhe. 
Corvus cornix wird nur im Winter gesehen.

Corvus corax. Von diesem horstete ein Pärchen bei Helmers, von dem sich das $\sigma$ in meiner Sammlung befindet. Das 오 wurde geflügelt und entkam.

Corvus frugilegus ist noch nicht beobachtet worden.

Oriolus galbula scheint ganz zu fehlen. Wenigstens hat noch Niemand, soviel ich erfahren habe, seinen bekannten Ruf vernommen.

Sturnus vulgaris zahlreich und besonders im Herbst in kolossalen Schwärmen im Weidengebüsch der Werra Nachtruhe haltend.

Turdus merula zahlreich bei Liebenstein und im Silbergrund.

Turdus musicus Brutvogel, häufig.

Cinclus aquaticus. Im Trusenthal häufig, auch an anderen Bächen.

Muscicapa grisola zahlreich und Brutvogel.

Ficedula hypolais und Sylvia hortensis ebenso.

Saxicola oenanthe nistet allenthalben in steinigen Hohlwegen.

Regulus ignicapillus sah ich zahlreich am Eisberg bei Witzelroda.

Motacilla alba sehr gemein.

Motacilla boarula im Trusenthal.

Alauda arvensis gemein.

Alauda cristata bemerkte ich nicht.

Parus major, coeruleus, cristatus, ater durchweg gemein.

Passer domesticus. Die weissen Sperlinge von Waldfisch sind in der ganzen Gegend bekannt. Ich selbst habe allerdings noch nicht das Glück gehabt, dort einen zu sehen. Dagegen sah ich einen solchen im Gebiet gefangen heim Besitzer des Gasthofes zum Hirsch in Liebenstein. Der Vogel hatte schwarze Augen und war im Uebrigen schneeweiss. Er machte einen etwas fremdartigen Eindruck, sobald er aber seine Stimme hören liess, war jeder Zweifel gehoben.

Passer montanus und Fringilla coelebs beide, namentlich letzterer sehr gemein.

Fringilla carduelis zahlreich im Herbst an den Chausséen.

Fringilla serina habe ich, wenigstens im Herbst, stets zahlreich angetroffen.

Fringilla chloris. An der Fischbrücke und bei Immelborn 
habe ich ihn häufig gehört und gesehen. Pyrrhula soll sich häufig bei Immelborn zeigen.

Emberiza citrinella zahlreich.

Emberiza miliaria und hortulana sind unbekannt.

Caprimulgus europaeus im Gebiet vorhanden.

Hirundo urbica und Hirundo rustica in allen Ortschaften sehr zahlreich. Für Hirundo riparia finden sich keine geeigneten Brutorte.

Certhia familiaris und Sitta europaea häufig (letztere vor dem Gasthofe in Wilhelmsthal sehr zutraulich).

Upupa epops nicht sehr häufig.

Alcedo ispida häufig an Gräben und besonders an der Werra.

Picus martius nistet in den Bergen. Ich erhielt ein 오 in der Mauser und ein junges Männchen, die beide todt gefunden worden waren.

Picus viridis und major beide gleich gemein.

Picus minor. Ein junges Exemplar erlegte ich bei Witzelroda in den Obstbäumen der Chaussée.

Jynx torquilla häufiger Brutvogel.

Cuculus canorus häufig. Die jungen Vögel im Herbst zeigen sich im Gegensatze zu den alten ganz und gar nicht scheu.

Columba oenas ist einer der häufigsten Vögel im Gebiet und in grossen Schaaren anzutreffen.

Columba turtur sah ich öfter, dagegen scheint $C$. palumbus $\mathrm{zu}$ fehlen.

Ardea cinerea sehr häufig.

Ciconia alba häufiger Brutvogel.

Ciconia nigra. Junge Vögel zeigten sich im Herbst 1879 auf den Werrawiesen. Einer wurde erlegt und mir überbracht. Ueber ein horsten im Gebiet konnte ich nichts ermitteln.

Scolopax gallinago zahlreich.

Vanellus cristatus am Frauenbreitunger See sah ich ihn zahlreich mit Anas boschas zusammen.

Fulica atra nistet zahlreich auf dem Forstloch bei Immelborn.

Gallinula chloropus und porzana sind beide auf den Teichen und Seen anzutreffen.

Crex pratensis häufig.

Podiceps minor erhielt ich einmal, das Exemplar hatte sich an den Telegraphendrähten der Werrabahn den Kopf einge- 
stossen. Das gleiche Schicksal hatten öfter auch die drei vorhergehenden Arten. Landvögel scheinen weniger dieser Gefahr ausgesetzt, wenigstens bemerkte ich nur einen jungen grauen Fliegenschnäpper nnter der Leitung.

Larus ridibundus soll am Frauenbreitunger See beobachtet worden sein.

Anser cinereus fast jeden Winter in zahlreichen Schwärmen an der Werra.

Anser torquatus. Im October vorigen Jahres wurde mir ein junger Vogel (vom Schreiner Schmidt in Barchfeld geschossen) zugesandt.

Anas boschas ist sehr zahlreich auf den Teichen und besonders auf dem Frauenbreitunger See.

Anas crecca. Wie vorige, aber weniger zahlreich.

Anas glacialis. Ein junges Weibchen wurde ebenfalls vom Schreiner Schmidt im Herbst vorigen Jahres erlegt und befindet sich in meiner Sammlung.

Mergus merganser soll sich in manchen Wintern auf dem Frauenbreitunger See blicken lassen.

\section{Die europäischen grossen Würger.}

\section{Von}

\section{E. F. von Homeyer.}

Herr Inspector Meves aus Stockholm hatte die Güte, mir behufs Vorlegung bei Gelegenheit der Versammlung der Deutschen Ornithologen zu Berlin 23 Stück schöner Bälge von Lanius excubitor und major $\mathrm{zu}$ senden. Ich verglich dieselben in Berlin mit den dortigen Exemplaren des Museums und später in Anclam mit der Sammlung des Herrn Tancré. Derselbe hatte die Güte, mir die 11 Exemplare seiner Sammlung zuzusenden, so dass ich mit meinen 17 Stück jetzt 51 dieser Würger zur Hand habe, um dieselben auf ihre Artbeständigkeit prüfen zu können, ohne alle die vielen Stücke in Betracht zu ziehen, welche ich früher zu sehen und zu vergleichen Gelegenheit hatte.

Schon bei meinen früheren Untersuchungen hatte ich starke Zweifel, ob es möglich sein würde, diese beiden Arten zu unterscheiden, indem der Armspiegel, der sich bei dem typischen Lanius excubitor als grosser weisser Fleck zeigt, bei einer Reihe 


\section{$2 \mathrm{BHL}$ Biodiversity Heritage Library}

1880. "Beitrag zur Ornithologie des Werrathales in Thüringen." Journal fu

r Ornithologie 28, 144-148. https://doi.org/10.1007/bf02008805.

View This Item Online: https://www.biodiversitylibrary.org/item/101708

DOI: https://doi.org/10.1007/bf02008805

Permalink: https://www.biodiversitylibrary.org/partpdf/142246

\section{Holding Institution}

Smithsonian Libraries

\section{Sponsored by}

Biodiversity Heritage Library

\section{Copyright \& Reuse}

Copyright Status: Public domain. The BHL considers that this work is no longer under copyright protection.

This document was created from content at the Biodiversity Heritage Library, the world's largest open access digital library for biodiversity literature and archives. Visit BHL at https://www.biodiversitylibrary.org. 